\title{
Does a New Information Structure about Cosmetic Outcome of Breast-Conserving Treatment Result in a Better Informed Patient? Outcome of a Comparative Case Study
}

\author{
ATPM Brands-Appeldoorn, MSc ${ }^{1^{*}}$, AJG Maaskant-Braat, MD, PhD ${ }^{1}$, VCG Tjan-Heijnen, \\ $M D, P h D^{2}$ and $R M H$ Roumen, MD, PhD ${ }^{1,2}$
}

${ }^{1}$ Department of Surgery, Máxima Medical Center, The Netherlands

${ }^{2}$ Division of Medical Oncology, GROW - School for Oncology and Developmental Biology, Maastricht, The Netherlands

*Corresponding author: Angelique (ATPM) Brands-Appeldoorn, MSc, Department of Surgery, Máxima Medical Center, Breast Unit, De Run 4600, P.O. Box 7777, 5500 MB Veldhoven, The Netherlands, Tel: +31-40-8886150, Fax: +31-408886159

\begin{abstract}
Background: To investigate whether the implementation of additional modern information tools about cosmetic outcome of breast-conserving treatment (BCT) could improve patient satisfaction.

Methods: A comparative case study compared data of two cross-sectional studies. The historical group (HG) was treated in 2013, the implementation group (IG) in 2016. The HG received a questionnaire, which resulted in implementation of the following items in our practice, expansion of the BCT brochure and photo book, incorporation of the Harvard and Numeric Rating scale. The IG received the same questionnaire, with the addition of nine more questions concerning specific implementation aspects.

Results: The HG contained 76 patients (age 41-86), versus 57 in the IG (age 40-84). Although additional information tools were implemented, no significant enhancement of IG patient satisfaction could be demonstrated. Nevertheless, the need for information appeared significantly greater $(p<$ 0.001) in the IG.

Conclusion: The need for information about cosmesis was greater in the IG, possibly due to increased attention paid by professionals. Furthermore, it is difficult to improve an information program concerning cosmetic consequences of BCT, despite the implementation of better tools. Professionals should focus on individual cosmetic outcome information during the entire treatment and follow up process of BCT.
\end{abstract}

\section{Keywords}

Breast Neoplasms, Breast-Conserving surgery, Cosmetic outcome, Cosmetic evaluation, Patient satisfaction

\section{Introduction}

Breast cancer is the most common malignancy in women in the Netherlands. Based on tumor characteristics and personal decision making the majority of these women will chose to undergo breast-conserving treatment (BCT). The principle of BCT consists of a wide local tumor excision and postoperative whole-breast irradiation. Goals of this treatment are to achieve an optimal local tumor control with a good cosmetic outcome [1]. However, breast deformation may occur later on $[2,3]$. Considering the incorporation of "shared decision making", it is important to discuss a possible breast deformation as a side-effect of BCT [4]. Timing of this sensitive information during the whole treatment pathway seems to be a relevant issue $[5,6]$. How and when do patients want to be informed about the cosmetic aspects of BCT? Currently, literature on the specific information needs of patients concerning the cosmetic effects of BCT is lacking. A number of studies investigated patient-information requirements of cancer patients in general, and assessed the effects of BCT on psychoso-

Citation: Brands-Appeldoorn ATPM, Maaskant-Braat AJG, Tjan-Heijnen VCG, Roumen RMH (2019) Does a New Information Structure about Cosmetic Outcome of Breast-Conserving Treatment Result in a Better Informed Patient? Outcome of a Comparative Case Study. Int J Cancer Clin Res 6:109. doi. org/10.23937/2378-3419/1410109

Accepted: April 15, 2019; Published: April 17, 2019

Copyright: (C) 2019 Brands-Appeldoorn ATPM, et al. This is an open-access article distributed under the terms of the Creative Commons Attribution License, which permits unrestricted use, distribution, and reproduction in any medium, provided the original author and source are credited. 
cial functioning and quality of life [5-11]. However, data on the specific needs for information regarding cosmetic effects of BCT are currently lacking. In 2013, patient experiences regarding their need for information of possible cosmetic effects following $\mathrm{BCT}$, were analysed in our hospital indicating a need for optimization of information in these breast cancer patients [12]. Based on their suggestions, we implemented a number of new information tools and analysed their impact on patient satisfaction.

Aim of the present study was to investigate whether the implementation of additional and more modern information tools about cosmetic outcome of BCT could improve patient satisfaction. It was hypothesized that implementing a number of new information tools would increase patient satisfaction on information.

\section{Methods}

This is a comparative case study about patient information needs concerning the cosmetic effects of BCT, comparing data of two cross-sectional studies including a historical setting.

\section{General patient information}

Two groups of patients treated for invasive breast cancer or Ductal Carcinoma In Situ (DCIS) with BCT at the breast clinic of Máxima Medical Center (MMC) were studied. MMC is a 614 bed teaching hospital in Veldhoven and Eindhoven, the Netherlands. In our breast clinic some 300 new patients with (invasive) breast cancer are seen yearly by certified breast surgeons and nurse practitioners.

The historical group consisted of patients who were treated in 2013, while the prospective control group was treated in 2016. In 2014 and 2017, respectively at least one year after the breast operation, patients were asked to participate in this study. Patients with a previous mastectomy or BCT of the contralateral breast, $B C T$ in combination with intraoperative radiotherapy, BCT including nipple removal, metastatic disease at presentation or during follow up, as well as patients with local recurrence or any (previous) plastic breast surgery were excluded.

The study was reviewed by the Medical Ethical Review board of MMC (date: 10-07-2012) confirming that the study did not require formal ethics approval. Data were collected anonymously and treated according to present applicable Dutch laws and in line with the Declaration of Helsinki [13].

\section{Study design}

First, patients treated in 2013 (historical group) were asked to evaluate the information they had received from the medical professionals (surgeons and nurse practitioners) about the cosmetic consequences of BCT and other information needs using a questionnaire consisting of 12 questions (Addendum 1). Next, based on the evaluation and results of this questionnaire, in 2015 the following items were implemented in our practice to optimize the information:

- The text of the information brochure about BCT was expanded by describing the possible cosmetic consequences and various reconstruction options, such as partial prosthesis or reconstructive plastic surgery.

- The Harvard scale and a Numeric Rating Scale for evaluation of cosmetic outcome were incorporated into the electronic patient file. Patients and medical professionals were asked to standardly give a score during the treatment pathway and follow up visits. The Harvard scale is the most widely used validated and subjective scoring system, categorizing patient's cosmesis into excellent, good, fair or poor [14].

- With participation of Dutch breast cancer patients a photo book was constructed showing various cosmetic results of BCT.

Next, patients treated in 2016 were evaluated using the same questionnaire (Addendum 2) as previously sent to the patients treated in 2013 , with the addition of nine questions concerning specific implementation aspects. These were called the implementation group.

\section{Statistical analysis}

The results of both questionnaires were processed using the online application 'Explora' of NVZ Plus (NVZ, 2017) [15].

Data were analysed using the Statistical Package for the Social Sciences (SPSS, SPSS Inc. Chicago, IL, US), version 22.

Descriptive statistics were used to describe baseline characteristics of the study population. Categorical variables were presented as numbers with frequencies. Continuous variables were presented as mean with standard deviation (SD) if normally distributed and median with range (minimum to maximum) if not normally distributed. Proportions were presented with 95\%-confidence interval where applicable.

Comparisons between populations before and after implementation of the new information protocols were statistically tested by applying Chi-square statistics for categorical variables and a Mann-Whitney $U$ test for not normally distributed continuous variables. In case of ordinal categorical variables we also did a trend test by calculating the linear-by-linear $p$-value. Since the intention of this study was mostly hypothesis generating, we did not correct for multiple testing and assumed a potential effect at a two-sided $p$-value of 0.05 .

\section{Results}

\section{General information}

The response rate to the questionnaires was $78 \%$ ( $n$ 
Table 1: Changes of various aspects of information on cosmetic outcome after BCT.

\begin{tabular}{|l|l|l|l|}
\hline Aspects of information & Historical group (\%) & Implementation group (\%) & p-value \\
\hline Information received & 71 & 83 & 0.10 \\
\hline Need for information & 18 & 68 & $<0.001$ \\
\hline Importance of information & 75 & 85 & 0.24 \\
\hline Satisfaction about attention during follow up & 81 & 88 & 0.44 \\
\hline
\end{tabular}

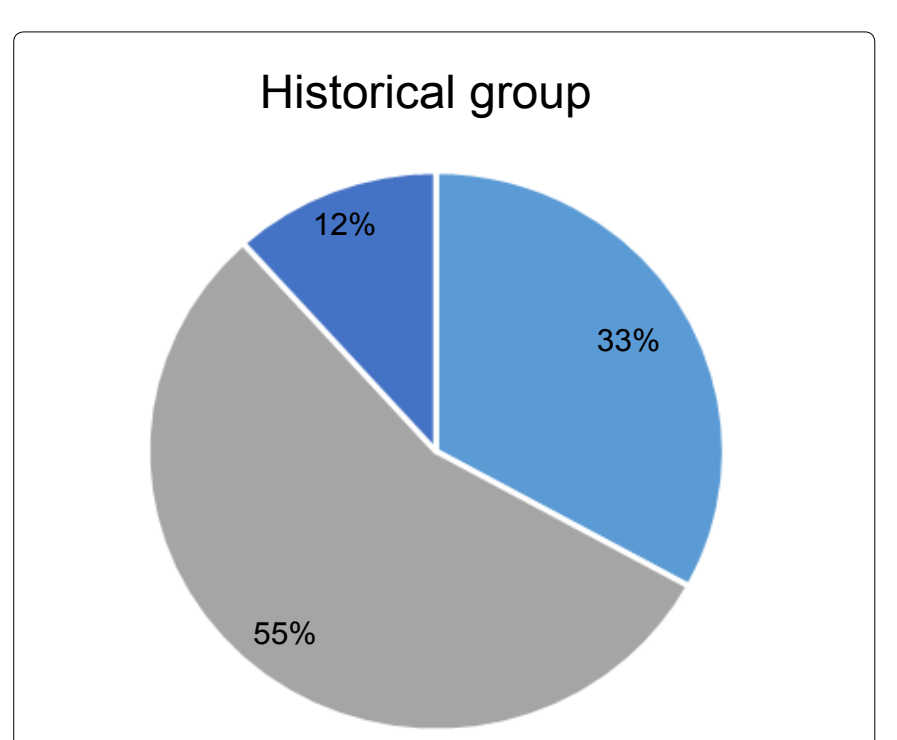

a surgeon a nurse practitioner a radiotherapist

Figure 1A: Patient informed by different disciplines about cosmetic outcome of BCT (historical group).

Implementation group

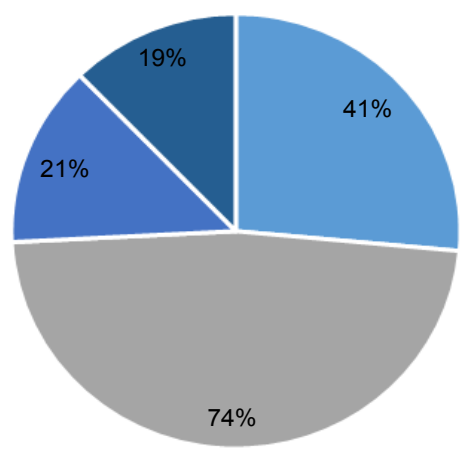

" surgeon = nurse practitioner = radiotherapist = unknown

Figure 1B: Patient informed by different disciplines about cosmetic outcome of BCT (implementation group).

$=76 / 98)$ in the historical group and $79 \%(n=60 / 76)$ in the implementation group respectively. The median age of the historical group was 66 years (range: $41-86$ years) and 64 years (range: $40-84$ years) in the implementation group.

The majority of patients in both groups scored the treated breast compared to the untreated breast as 'no or only a small difference': $82 \%(n=60)$ in the historical group versus $85 \%(n=51)$ in the implementation group $(P=0.98)$, respectively. There was no significant difference between both groups regarding satisfaction of cosmetic outcome: $65 \%(n=48)$ in the historical group versus $77 \%(n=46)$ in the implementation group $(P=0.39)$.

\section{Educational material}

Table 1 shows changes of various aspects of information on cosmetic outcome after BCT. $71 \%$ of the patients in the historical group indicated that they had received information about cosmetic outcome before or around treatment versus $83 \%$ of the patients in the implementation group $(P=0.10)$. Noteworthy, there was a significant difference between both groups regarding the need for information about cosmetic effects: $68 \%$ $(n=40)$ in the implementation group versus $18 \%$ ( $n$ $=8)$ in the historical group $(P<0.001)$. Figure $1 \mathrm{~A}$ and Figure $1 \mathrm{~B}$ show that the nurse practitioner has got an increasingly prominent role in informing patients about the cosmetic outcome of BCT. There was no significant difference $(P=0.79)$ in degree of satisfaction of the given information about BCT using a numeric rating scale in both groups (Figure 2). The mean numeric score in the historical group was 8.1 (SD 1.1) versus 7.8 (SD 1.7) in the implementation group.

The timing at which patients prefer to receive information was comparable for both groups, being before operation and during follow up visits for the majority of patients and postoperatively or after radiotherapy for the minority. Patients in both groups $(75 \%$ in the historical group versus $85 \%$ in the implementation group) found it very important that the cosmetic outcome was discussed with them prior to treatment $(P=0.24)$. There was no statistical significance $(P=0.44)$ between both groups regarding the satisfaction of the attention that was given to the cosmetic outcome during follow up: $81 \%$ of the patients in the historical group versus $88 \%$ of the patients in the implementation group found that enough attention had been paid. Both groups indicated that this is an important issue in this part of the treatment process, since the treated breast can change over the years.

\section{Ways of information and patient satisfaction}

Prior to implementation, the historical patient group was asked about which methods of information on the cosmetic outcome of BCT they would prefer (Table 2). Patients in this group particularly had the need for oral information (87\%), followed by written (26\%), visual (17\%) and digital (20\%) information. Most of the 


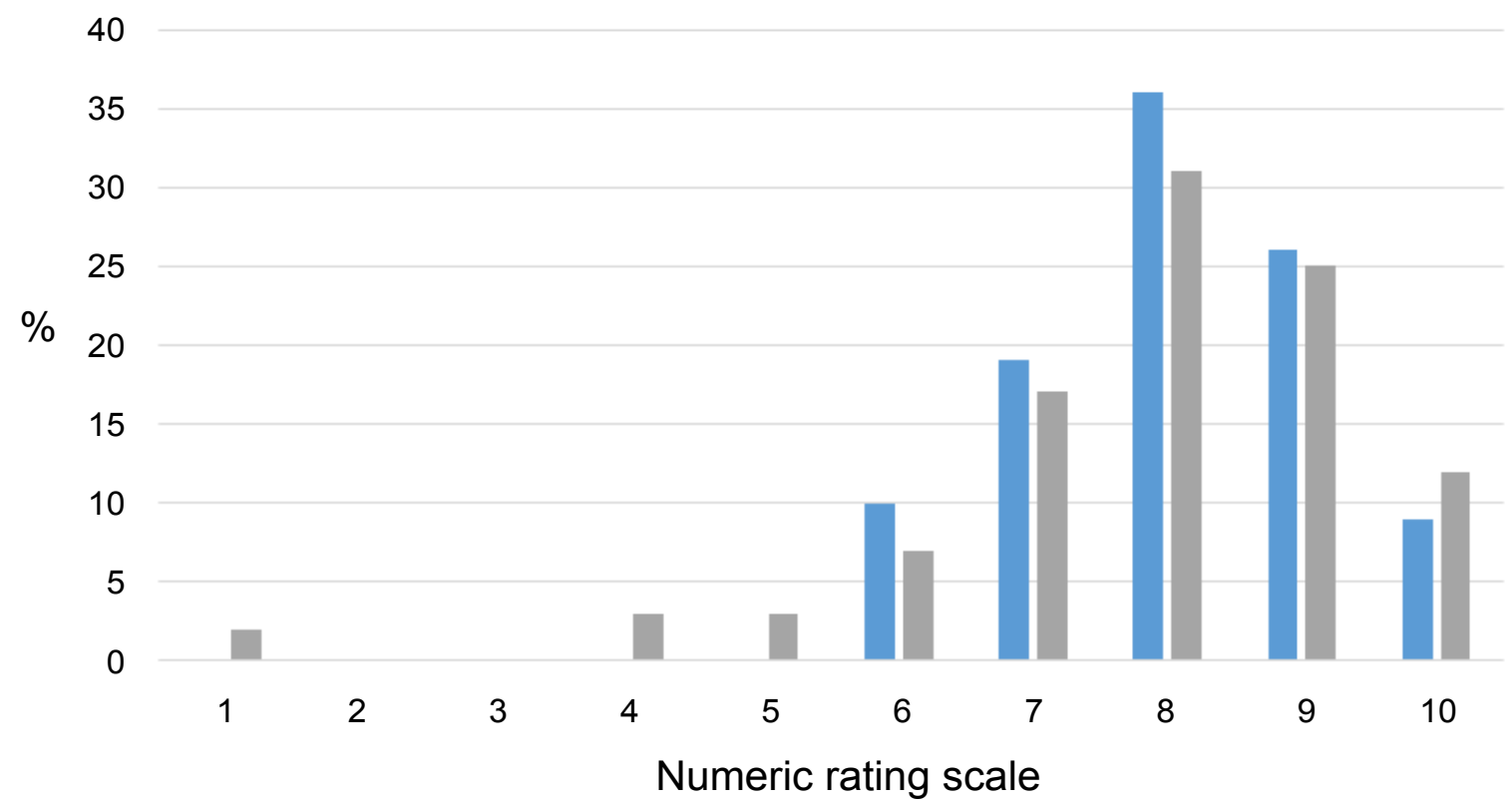

historical group $(\mathrm{n}=53) \quad$ implementation group $(\mathrm{n}=59)$

Figure 2: Assessment of the given information about cosmetic effects of BCT using a numeric rating scale $(1=$ poor, $10=$ excellent).

Table 2: Way of information needs historical group (multiple answers possible).

\begin{tabular}{|l|l|}
\hline Kind of information & $(\mathbf{n}=\mathbf{7 5 )}(\mathbf{\% )}$ \\
\hline Oral & $66(86.6)$ \\
\hline Written & $20(26.3)$ \\
\hline Visually (e.g. photobook) & $13(17.1)$ \\
\hline Digital (e.g. website) & $15(19.7)$ \\
\hline
\end{tabular}

patients in the implementation group had received oral $(83 \%)$ and written (88\%) information. $81 \%$ of the patients was satisfied about the oral information. Patients indicated that they were told that the treated breast could change over the years. The majority of the patients $(74 \%)$ were satisfied about the received written information. They found the information flyer about the cosmetic consequences of BCT clear and complete. The photo book with examples of cosmetic results after BCT has been seen by only $19 \%$ of the patients and they were satisfied with it. Some patients (other 19\%) indicated that they did not have the need for this specific information. Some mentioned that they found the pictures too confronting or that at that moment issues of survival of breast cancer had the priority. However, $54 \%$ of the patients noted that a look at the photo book was not offered to them by the nurse practitioner.

\section{Information during follow up}

Forty-nine percent of the patients $(95 \% \mathrm{Cl} 36-62)$ in the implementation group indicated that during the follow up visits the professional had asked them to give their opinion and score for the appearance of the treated breast. Sixty-one percent (95\% Cl 48-74) found that asking about this matter, made the cosmetic consequences of BCT easier to discuss. Because more attention has been paid to the cosmetic effects, patients indicated that during the follow up visits alternative possibilities were discussed (for example partial prosthesis, reconstructive operations). In the historical group, $13 \%$ of the patients indicated that these possibilities were not discussed with them, although they felt a need for it, versus $8 \%$ in the implementation group.

\section{Discussion and Conclusion}

\section{Discussion}

In this current study we investigated whether the implementation of additional and more modern information tools about cosmetic outcome of BCT could improve patient satisfaction. Although various changes in this respect were implemented, we could not demonstrate a statistically significant enhancement of patient satisfaction between the historical and implementation group, albeit that we saw a trend of improvement on various aspects.

Notably, the need for information about the cosmetic consequences in the implementation group in comparison with the historical group was significantly higher. Possibly, the emphasis that we put on this issue and thereby increasing awareness about this cosmetic subject, led to an increase in the implementation group. This can be seen as some kind of Hawthorne effect [16], being the effect of an intervention on a study participant that is only due to the fact that there is some study participation. Priority for our study patients 
was healing and survival. Later but also during various different phases of treatment, they wanted to receive information about possible cosmetic consequences of the treatment from their attending doctor and nurse practitioner. These findings are consistent with literature [6].

More patients from the implementation group indicated that they had received information about the cosmetic outcome compared to the historical group (83 versus $71 \%$ ). We had hoped that by improving the information structure about cosmetic consequences, all patients would be informed about this. Although it can be possible that in daily practice, let's say $5 \%$ of patients may not have received the standard information package, still more than $10 \%$ did not recall having received information 1 year after they actually did.

In both groups the quality of the given information was rated equally using a numeric rating scale. We had presumed that after the implementation phase the quality of care would have been graded higher, but we were not able to confirm this. In the historical group there was a need to get cosmetic information especially in an orally and written way. The addition of written information as an extension on this issue apparently did not result in a higher satisfaction level for these patients. Unfortunately, most of the patients in the implementation group did not receive the visual information provided by the newly designed photo book. Of those who have seen the photo book, the majority was satisfied. Others had no need for this specific book.

Discussion and visualization of the cosmetic effects in the different phases of treatment will lead to realistic expectations by patients. Ultimately, it might become easier for patients to accept a possibly worse cosmetic result and to make the wearing of a partial prosthesis or plastic surgical intervention negotiable [11]. This whole process of information and guidance to come to the right decision during the whole treatment phase is called "shared decision making" [4]. The present data show that by asking patients during follow up about their own opinion regarding the cosmetic aspects, this results in more attention for this topic. We did this by asking to give a numeric rating and application of the Harvard score [14]. By doing so, it becomes easier for patients to discuss the cosmetic consequences. Although, compared to the historical situation in which this was no routine, in the structured implementation period this was done in about halve of the cases. Despite the fact that the majority of patients in this study were satisfied with the cosmetic outcome itself, continuous attention should be paid to the whole group of patients, thus including those who potentially are not satisfied. Therefore we conclude that there is still space for improvement and instructions for members of the whole breast cancer team.

At present, "Patient Reported Outcome Measures
(PROMs)" are considered of the utmost importance, fitting very well within the concept of the development of Value Based Health Care [17]. The value of care is the highest priority and the quality is determined by the patient herself. Our current data show that new implementations are a difficult process. In this present study, two different groups of patients were questioned and evaluated during different time frames and the need for information about the cosmetic outcome appeared to be different. In daily practice we would prefer to focus information on individual needs.

Although in literature specific information on patients' needs for information about the cosmetic effects of BCT in particular is lacking, results from various studies about information needs of cancer patients in general, are very similar to the results of the current study $[5,6,8,10,13]$. Patients indicated that they would like to receive oral, written, visual and digital information about the cosmetic consequences which is in line with previous studies [5,6,8,10,13].

\section{Limitations}

The current study shows that implementation of the presentation of the newly designed photo book concerning various cosmetic outcomes of BCT was not successful. Most of the patients indicated that this specific photo book was not presented to them, while patients from the baseline group indicated that there was a great need for such a tool. Because of this, the result of this specific implementation could not properly be assessed. Furthermore, this outcome shows that this tool is not offered structurally in the treatment pathway.

Another limitation of the present study is that it was difficult to assess the external validity, because the present study was performed in a single centre with a small sample size. Nevertheless, we think that this comparative case study gives a clear overview about breast cancer patient cosmetic information needs.

\section{Conclusion}

This study shows that it is difficult to improve an information program concerning cosmetic consequences of BCT, despite the implementation of better tools. Although the majority of patients indicated they were satisfied about cosmesis, the need for information on this issue was greater in the implementation group. This was possibly due to the increased attention on cosmetic outcome paid by the professionals or tendency of present time in which side effects of treatment will become a more important issue. Generally, not all patients want to discuss information about possible side effects (in this case, cosmetic consequences) at the same moment during the treatment process. So, professionals should focus on individual need on cosmetic outcome information during the entire treatment and follow up process of BCT. 


\section{Funding}

The authors declare that they have no sources of funding.

\section{References}

1. NABON (2012) Guideline Breast Cancer 2012.

2. Beadle GF, Silver B, Botnick L, Hellman S, Harris JR (1984) Cosmetic results following primary radiation therapy for early breast cancer. Cancer 54: 2911-2918.

3. Hennigs A, Hartmann B, Rauch G, Golatta M, Tabatabai P (2015) Long-term objective esthetic outcome after breast-conserving therapy. Breast Cancer Research and Treatment 153: 345-351.

4. Elwyn G, Frosch D, Thomson R, Joseph-Williams N, Lloyd A, et al. (2012) Shared Decision Making: A Model for Clinical Practice. J Gen Intern Med 27: 1361-1367.

5. Van der Kruk T (1998) Niet iedere patiënt wil overal over meebeslissen [Not every patient wants a say about everything]. TVZ TijdschriftvoorVerpleegkundigen 12: 375377.

6. Van Eenbergen M, Van Driel H, Van de Poll L (2006) Patiënt met kanker wil specialist met website. [Cancer patient wants specialist website]. Oncologica 4: 34-36.

7. Ciammella $P$, Podgornii $A$, Galeandro $M$, Micera $R$, Ramundo D, et al. (2014) Toxicity and cosmetic outcome of hypofractionated whole-breast radiotherapy: Predictive clinical and dosimetric factors. Radiat Oncol 9: 97.

8. Luker KA, Beaver K, Leinster SJ, Owens RG, Degner LF, et al. (1995) The information needs of women newly diagnosed with breast cancer. J Adv Nurs 22: 134-141.
9. Olivotto IA, Whelan TJ, Parpia S, Kim DH, Berrang T, et al. (2013) Interim cosmetic and toxicity results from RAPID: A randomized trial of accelerated partial breast irradiation using three-dimensional conformal external beam radiation therapy. J Clin Oncol 31: 4038-4045.

10. Van Vliet M, Grypdonck M, Van Zuuren F, Winnubst J, Kruitwagen C (2004) Psychologische voorbereiding op een gastroscopie: de invloed van informatie in medische situaties. Verpleegkunde 19: 196-207.

11. Waljee JF, Hu ES, Ubel PA, Smith DM, Newman LA (2008) Effect of esthetic outcome after breast-conserving surgery on psychosocial functioning and quality of life. J Clin Oncol 26: 3331-3337.

12. Brands-Appeldoorn A, Maaskant-Braat S, Schenk K, Roumen R (2018) Cosmetic consequences of breastconserving treatment for breast cancer: something worth talking about. Oncologist 23: 1-6.

13. World Medical Association (2013) World Medical Association Declaration of Helsinki: Ethical Principles for Medical Research Involving Human Subjects. JAMA 310: 2191-2194.

14. Harris JR, Levene MB, Svensson G, Hellman S (1979) Analysis of cosmetic results following primary radiation therapy for stages I and II carcinoma of the breast. Int J Radiat Oncol Biol Phys 5: 257-261.

15. NVZ (2017) Explora.

16. https://en.wikipedia.org/wiki/Hawthorne_effect

17. Porter ME (2010) What is value in health care? The New England Journal of Medicine 363: 2477-2481. 


\section{Addendum 1}

\section{Structured patient questionnaire historical group}

1. What is, in your own opinion, the difference between the treated breast compared to the untreated breast?

○ no difference

○ a small difference

○ a moderate difference

$\circ$ a large difference

2. How satisfied are you with the appearance of your treated breast compared to the untreated breast?

o very satisfied, the treated breast is almost identical to the untreated breast

- satisfied, the treated breast is only a little bit different from the untreated breast

- not unsatisfied, the treated breast is clearly different from the untreated breast, but not seriously deformed

○ dissatisfied, the treated breast is seriously deformed

3. Did you get any information on the cosmetic consequences of the treatment before or during treatment?

O yes, continue to question 4

o no, continue to question 6

4. Who discussed this with you and/or provided you with information?

○ surgeon

o yes

○ no

o surgeon in training

$\circ$ yes

no

- nurse practitioner

$\circ$ yes

o no

$\circ$ radiotherapist

$\circ$ yes o no

o otherwise, namely.

5. Could you grade the information you have received by the medical specialist/nurse practitioner about the cosmetic outcome of breast conserving treatment (even if this was hardly or not the case!)? 1 = very bad, $10=$ excellent.

○ 1

$\circ 2$

○ 3

○ 4

$\circ 5$

○ 6

$\circ 7$

○ 8

$\circ 9$

० 10

6. Did you or would you have a need for information about the cosmetic consequences of breastconserving treatment?

- yes, I would have liked information about the possibility of deformation of the treated breast as a result of the treatment

- no, at the moment of diagnosis I was not concerned with possible cosmetic changes of the treated breast

o otherwise, namely.

7. Did the medical specialist/nurse practitioner pay attention to the cosmetic consequences of breastconserving treatment during the follow-up visits?

$\circ$ yes, sufficient attention

$\circ$ yes, but insufficient attention

o no, no attention paid at all

8. Please indicate at which stage of your treatment you would like to receive information about the cosmetic consequences of the treatment? (Multiple answers possible)

before the operation

yes

no

about 2 weeks after the operation. 
o yes
o no

○ about 1 month after the operation

○ yes

o no

o during follow-up checks

○ yes

○ no

9. Please indicate how important it is to you that the medical specialist/nurse practitioner discusses the cosmetic consequences of the treatment with you prior to surgery?

- very important (high priority)

○ important (average priority)

○ not important (low priority)

10.The treated breast may become deformed over the years. Would you like to indicate how important it is to you that the medical specialist/nurse practitioner discusses the cosmetic consequences of the treatment during the follow-up visits?

○ very important (high priority)

○ important (average priority)

○ not important (low priority)

11.In what way would you like to receive information about the possible cosmetic consequences of breastconserving treatment? (Multiple answers possible)

o oral information by medical specialist/nurse practitioner

○ yes

○ no

o written information, such as by a patient information flyer

○ yes

o no

○ visual information (for example a photo book)

○ yes

○ no

○ digital information (for example a website)

○ yes

o no

12.Do the medical specialist/nurse practitioner discuss various options like partial prosthesis or reconstructive operations during the follow up visits in case you indicate that you are not satisfied with the cosmetic outcome of the treated breast?

○ yes

o no, but I would like to get such information in case my treated breast may start to deform

- no, I have no interest in such information

○ inapplicable 


\section{Addendum 2}

\section{Structured patient questionnaire implementation group}

1. What is, in your own opinion, the difference between the treated breast compared to the untreated breast?

○ no difference

- a small difference

- a moderate difference

- a large difference

2. How satisfied are you with the appearance of your treated breast compared to the untreated breast?

- very satisfied, the treated breast is almost identical to the untreated breast

O satisfied, the treated breast is only a little bit different from the untreated breast

o from the untreated breast, but not seriously deformed

○ dissatisfied, the treated breast is seriously deformed

3. Did you get any information on the cosmetic consequences of the treatment before or during treatment?

o yes, continue to question 4

o no, continue to question 6

4. Who discussed this with you and/or provided you with information?

(multiple answers possible)

○ inapplicable, you answered question 3 with 'no'

○ surgeon

○ nurse practitioner

○ radiotherapist

5. Could you grade the information you have received by the medical specialist/nurse practitioner about the cosmetic outcome of breast conserving treatment (even if this was hardly the case!)? 1 = very bad, 10 = excellent.

$\circ 1$
$\circ \quad 2$
$\circ \quad 3$
○ 4

○ 5

○ 6

$\circ 7$

$\circ 8$

$\circ 9$

○ 10

6. Did you or would you have a need for information about the cosmetic consequences of breastconserving treatment?

○ yes

o no

7. At what point during the course of treatment did you need information about the cosmetic consequences of breast-conserving treatment?

○ before operation

o approximately 2 weeks after the operation, during the appointment in which the outcome of the operation and the further treatments were discussed

o approximately 1 month after the operation, during the appointment about aftercare with the nurse practitioner

o during the follow-up checks

o inapplicable, you answered question 6 with 'no'

8. Please indicate how important it is, in your opinion, that the medical specialist/nurse practitioner discusses the cosmetic consequences of the treatment prior to surgery?

○ very important (high priority)

○ important (average priority)

○ not important (low priority)

9. In your opinion, who should discuss the information about the possible cosmetic consequences of breastconserving treatment with the patient prior to surgery?

○ surgeon

○ nurse practitioner

○ both

10.Did the medical specialist/nurse practitioner pay attention to the cosmetic consequences of breast- 
conserving treatment during the follow-up visits?

$\bigcirc$ yes, sufficient attention

- yes, but insufficient attention

o no, no attention at all

Please, elaborate on your

answer:

11. The treated breast may become deformed over the years. Would you like to indicate how important it is to you that the medical specialist/nurse practitioner discusses the cosmetic consequences of the treatment during the follow-up visits?

o very important (high priority)

o important (average priority)

○ not important (low priority)

12. Did you receive oral information about possible cosmetic consequences of the treatment?

$\circ$ yes

o no

13. How satisfied are you with the content of this oral information?

o inapplicable, you answered question 12 with 'no'

○ very satisfied

○ satisfied

- reasonably satisfied

○ badly satisfied

Please, elaborate on your answer:

14. Did you receive the information brochure 'breastconserving surgery'?

$\circ$ yes

no

15. How satisfied are you with the information about possible cosmetic consequences of the treatment provided in this brochure?

o inapplicable, you answered question 14 with 'no'

$\circ$ very satisfied

○ satisfied
○ reasonably satisfied

O badly satisfied

Please, elaborate on your answer:.

16. Did you together with your nurse practitioner view the photo book concerning results of breastconserving treatment during treatment or followup?

○ yes

o no

17. How satisfied are you with the information about possible cosmetic consequences of the treatment by the photo book?

- inapplicable, you answered question 16 with 'no'

○ very satisfied

○ satisfied

○ reasonably satisfied

$\circ$ badly satisfied

Please, elaborate on your answer:

18. Can you indicate why you did not view the photo book with the results of breast-conserving treatment during treatment or follow-up?

o inapplicable, you answered question 16 with 'yes'

- I did not need this

$O$ is not offered by the nursing specialist

Please, elaborate on your

answer:

19. Were you asked by your medical specialist/nurse practitioner to give a numeric rating score concerning the cosmetic result of your treated breast during follow up visits?

o yes

o no

20. Do you feel that by asking your opinion about the appearance of your treated breast with some kind of a score, the cosmetic consequences of the treatment are easier to discuss?

○ yes

○ no 
Please, elaborate on your answer:

21.Do the medical specialist/nurse practitioner discuss various options like for example, partial prosthesis or reconstructive operations, in case you indicate that you are not satisfied with the cosmetic outcome of the treated breast? o inapplicable, I am satisfied with the appearance of the treated breast

o yes

o no, but I would like to get such information in case my treated breast may start to deform

o otherwise, namely. 